Vergleichung der Beobachtungen mit den Ephemeriden des Berl. Jahrb. rgo8 (B-R):

\begin{tabular}{|c|c|c|c|}
\hline I 9 & & $\Delta \alpha$ & $\Delta \delta$ \\
\hline & & (I 7 ) The & \\
\hline Jan. & 22 & $-23^{s} \cdot 64$ & $+I^{\prime} 8.0$ \\
\hline & 23 & -23.99 & + I 7.0 \\
\hline & 24 & -24.08 & + I 6.9 \\
\hline & & r9) Fort & \\
\hline Dez. & 7 & +18.72 & -5.5 \\
\hline & 22 & +18.75 & $-\quad 0.7$ \\
\hline & & 24) Ther & \\
\hline Okt. & I 4 & -0.81 & -3.0 \\
\hline & I 4 & -0.59 & -3.1 \\
\hline & & 5) Proser & \\
\hline Sept. & 8 & -3.30 & - r 9.8 \\
\hline & Io & -3.08 & -22.0 \\
\hline & I I & $-3.2 \mathrm{I}$ & -20.6 \\
\hline & & 28) Bell & \\
\hline Juni & 20 & +9.07 & -4.3 \\
\hline & 26 & +9.01 & $-5 \cdot 3$ \\
\hline Juli & I 2 & $+8.9^{2}$ & -8.0 \\
\hline & & (37) Fid & \\
\hline Juli & 12 & $+0.2 \mathrm{I}$ & -3.4 \\
\hline & I 7 & -0.15 & -0.1 \\
\hline & 20 & +0.02 & -4.8 \\
\hline
\end{tabular}

\begin{tabular}{|c|c|c|}
\hline I 906 & $\Delta \alpha$ & $\Delta \delta$ \\
\hline \multicolumn{3}{|c|}{ (47) Aglaja. } \\
\hline Sept. 24 & +0.66 & $+4:$ \\
\hline Okt. 9 & $+\quad 1.24$ & +7 \\
\hline
\end{tabular}

(57) Mnemosyne.

Jan. $23\left|-2^{\mathrm{m}} 45^{5} \cdot 7 \mathrm{I}\right|+6^{\prime}$ I $7^{\prime \prime}: 0$ $24|-245.74|+616.9$

(65) Cybele.

\begin{tabular}{rr|l|ll} 
Mai & 23 & -16562 & +1 & 20.5 \\
& 25 & -16.61 & $+I$ & 11.9
\end{tabular} (68) Leto.

\begin{tabular}{ll|l|l} 
Jan. I & +10.21 & -42.4 \\
& I 7 & +10.18 & -42.9 \\
22 & +10.40 & -41.0
\end{tabular}

(92) Undina.

Mai $28|-4.80|+24.2$

(I 13$)$ Amalthea.

Dez. $7|+1.18|+4.5$ \begin{tabular}{r|r|r}
7 & +1.15 & +4.9 \\
18 & +1.23 & +4.4
\end{tabular}

\begin{tabular}{|c|c|c|}
\hline r906 & $\Delta \alpha$ & $\Delta \delta$ \\
\hline \multicolumn{3}{|c|}{ (I 18) Peitho. } \\
\hline Jan. 24 & $-47^{5} \cdot 37$ & $+3^{\prime} 40^{\prime \prime} 6$ \\
\hline & -48.69 & +334.0 \\
\hline Febr. I 4 & -49.86 & +311.7 \\
\hline
\end{tabular}

(r34) Sophrosyne.

\begin{tabular}{rr|r|rr} 
Okt. 8 & -12.73 & $-\mathrm{I}$ & 4.5 \\
& 9 & -12.74 & $-\mathrm{I}$ & 5.5 \\
$\mathrm{IO}$ & -12.60 & $-\mathrm{I}$ & 6.4
\end{tabular}

(24 I) Germania.

\begin{tabular}{rr|r|r} 
Nov. I0 & -0.42 & +0.5 \\
20 & -0.35 & +1.9 \\
Dez. I8 & -0.13 & +1.7
\end{tabular}

(247) Eukrate.

Dez. \begin{tabular}{r|r|r}
7 & -3.67 & +6.8 \\
8 & -3.73 & +6.0 \\
$\mathrm{I} 8$ & -3.78 & +2.7 \\
$2 \mathrm{I}$ & -3.55 & +3.1
\end{tabular}

Diese 66 Beobachtungen von 24 Planeten, sowie die durch die hohe Deklination bedingten ungewöhnlich starken Anschlußbeobachtungen der schwachen Vergleichsterne 14, Phasenwirkung der Planet (247) Eukrate in unmittelbarer Nähe I 7 und 54, habe ich am Ringmikrometer nach der Augeund Ohr-Methode angestellt. Herrn Prof. E. C. Pickering danke ich auch an dieser Stelle bestens für die gütige Mitteilung der elf Sternpositionen aus den Harvard Zonen. Erwähnt sei noch, daß nach meinen Beobachtungen infolge der der Opposition 1906 eine Lichtschwächung im Betrage von 0.5 Größenklassen erfuhr. Es liegt demnach einer der seltenen Fälle vor, wo die übliche Formel $m=g+5(\log r+\log \Delta)$ auch für die Zeit der Opposition zu kleine Werte liefert, wenn Düsseldorf, Sternwarte, I 907 Januar 16.

Wilhelm Luther.

Cometa Finlay $1906 \mathrm{~d}$.

Equatoriale di Amici ad Arcetri. Obiettivo $284 \mathrm{~mm}$. Micrometro a lamine Ig"45. Ingr. I 24.

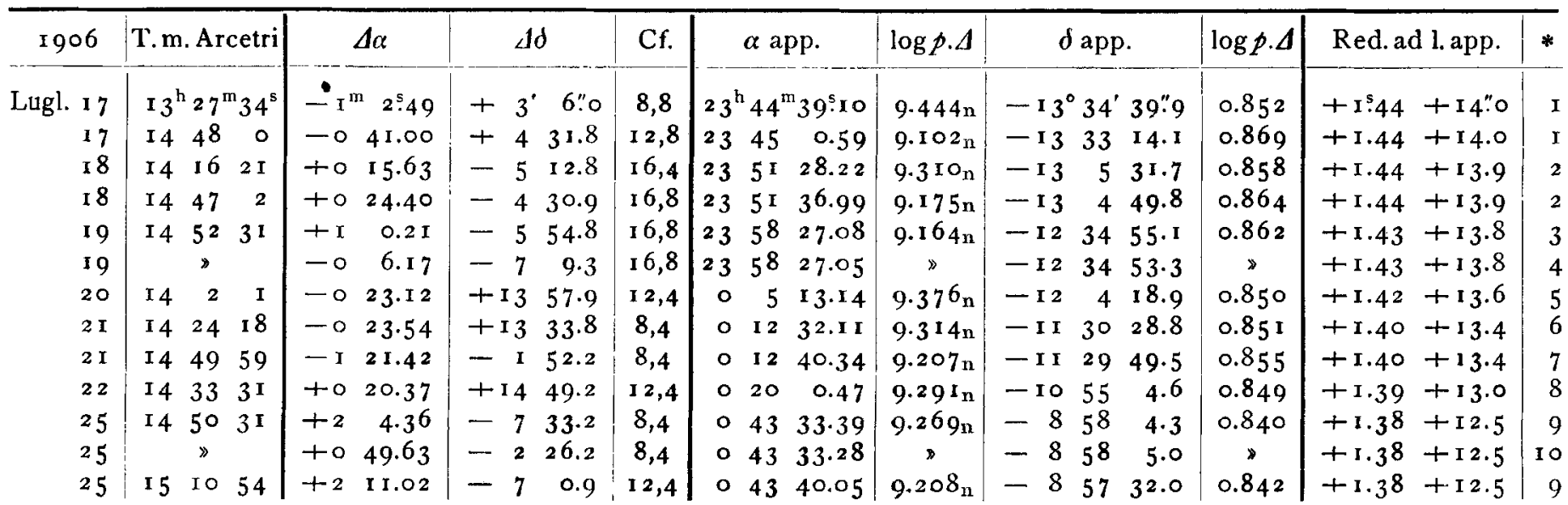




\begin{tabular}{|c|c|c|c|c|c|c|c|c|c|c|c|c|c|c|c|c|}
\hline \multirow{2}{*}{$\frac{1906}{\text { ugl. } 25}$} & T. m. Arcetri & $\Delta \alpha$ & \multicolumn{3}{|c|}{$\Delta \delta$} & \multirow{2}{*}{$\frac{\text { Cf. }}{12,4}$} & \multicolumn{2}{|r|}{$\alpha$ app. } & \multirow{2}{*}{$\begin{array}{l}\log p \cdot \Delta \\
9.208_{\mathrm{n}}\end{array}$} & \multicolumn{3}{|c|}{$\delta$ app. } & \multirow{2}{*}{$\frac{\log p . \Delta}{0.842}$} & \multicolumn{2}{|c|}{ Red. ad 1. app. } & \multirow{2}{*}{$\frac{1}{10}$} \\
\hline & $15^{\mathrm{h}} 10^{\mathrm{m}} 54^{\mathrm{s}}$ & $+o^{m} 5^{6}$ & - & & 53.5 & & & $43^{m} 39$ & & -8 & $8^{\circ} 57$ & & & $+I^{5} \cdot 3^{8}$ & +22.5 & \\
\hline 26 & I 45137 & to 16.65 & -1 & & $45 \cdot 3$ & 8,4 & $\circ 5$ & 5145.18 & $9.28 \mathrm{I}_{\mathrm{n}}$ & -8 & 815 & 35.6 & $0.83^{6}$ & +1.36 & +12.1 & I I \\
\hline 26 & 15448 & to 21.23 & -1 & & 22.9 & 8,4 & & $51 \quad 49.76$ & $9.227 \mathrm{n}$ & -8 & $8 \quad 15$ & 13.2 & .837 & +1.36 & +12.1 & I I \\
\hline 29 & 141922 & +216.27 & -1 & & 9.0 & 8,8 & I I & 179.43 & $9.419 \mathrm{n}$ & -5 & $5 \quad 59$ & I I . I & 0.818 & +1.33 & +11.1 & 12 \\
\hline 3 I & $14 \quad 1945$ & $\begin{array}{ll}-3 & 5.43\end{array}$ & -1 & 0 & 32.2 & 8,4 & I 3 & $34 \quad 54.09$ & $9.439 \mathrm{n}$ & -4 & 420 & 9.0 & 0.809 & +1.29 & +10.3 & I 3 \\
\hline 31 & 145236 & $-2 \quad 5^{2.74}$ & - & 9 & 21.7 & 8,4 & x 3 & $35 \quad 6.78$ & $9 \cdot 35 \mathrm{In}_{\mathrm{n}}$ & -4 & $4 \quad 18$ & 58.5 & 0.812 & $+\mathrm{I} .29$ & +10.3 & 13 \\
\hline os. I & 141543 & + I 35.89 & + & 5 & 53.6 & 8,4 & I 4 & $43 \quad 54.76$ & $9.459 \mathrm{n}$ & -3 & 329 & 1.1 & 0.805 & +1.28 & +9.8 & I 4 \\
\hline $\mathbf{I}$ & $》$ & +I 6.67 & - & 6 & 22.5 & 8,4 & I 4 & $43 \quad 54.63$ & $\bowtie$ & -3 & 329 & 0.4 & $»$ & $+\mathrm{I} .28$ & +9.8 & I 5 \\
\hline $\mathbf{I}$ & $15 \quad 16 \quad 34$ & + I $58.5^{\circ}$ & + & 8 & 1.7 & 8,4 & I 4 & $44 \quad 17.37$ & $9.29^{2} \mathrm{n}$ & -3 & 326 & 53.0 & .809 & +1.28 & +9.8 & I 4 \\
\hline $\mathbf{I}$ & $\gg$ & +129.24 & - & 4 & 12.9 & 8,4 & I 4 & $44 \times 7.20$ & 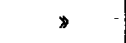 & -3 & 326 & 50.8 & $»$ & $+\mathrm{I} .28$ & +9.8 & I 5 \\
\hline 2 & I $5 \quad 924$ & to 28.53 & + & $\circ$ & 18.0 & 8,4 & I 5 & 5321.15 & $9 \cdot 33^{2} n$ & -2 & 234 & 55.2 & 0.803 & +1.25 & +9.3 & I 6 \\
\hline 2 & $\nabla$ & - 018.79 & - & $\mathbf{I}$ & 9.1 & 8,4 & & 5321. & 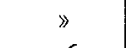 & -2 & 234 & 5 & $\gg$ & .25 & +9.3 & I 7 \\
\hline 2 & I $5 \quad 2843$ & to 13.98 & - & 3 & 20.3 & 8,4 & & $53 \quad 27.87$ & $9.264 n$ & -2 & 34 & 17.8 & 0.802 & $+\mathrm{I} .25$ & +9.3 & 18 \\
\hline 2 & 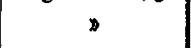 & -0 I 2.00 & - & 0 & 28.9 & 8,4 & I 5 & 5328.12 & 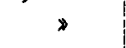 & -2 & 234 & 17.5 & $»$ & +1.25 & +9.3 & I 7 \\
\hline 3 & 151757 & to 17.00 & + & 2 & 48.6 & 24,8 & & - & $9 \cdot 3^{22} \mathrm{n}$ & & - & & 0.796 & +1.23 & +8.7 & I9 \\
\hline I 3 & $1428 \quad 48$ & -o 38.83 & - & 6 & $x 7 \cdot 5$ & 8,4 & 33 & $3^{2} \quad 5.45$ & $9.543 n$ & +6 & $65 \mathrm{I}$ & 32.0 & 0.748 & +I.II & +3.6 & 20 \\
\hline I 3 & $15 \quad 18 \quad 32$ & -021.81 & - & 4 & 37.6 & 8,4 & 33 & $32 \quad 22.47$ & $6_{n}$ & +6 & $5 \quad 53$ & 11.9 & 0.739 & + I.II & +3.6 & 20 \\
\hline 14 & $1532 \quad 21$ & $-2 \quad 0.96$ & - & 0 & 3.8 & 12,4 & 34 & 4050.10 & $7 \mathrm{n}$ & +7 & 739 & 1.9 & .730 & +1.10 & +3.2 & $2 \mathrm{I}$ \\
\hline 14 & 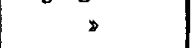 & $\begin{array}{ll}-2 & 2.5\end{array}$ & + & I & 3 . & 12,4 & 34 & $40 \quad 49$ & 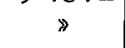 & +7 & 39 & 6.1 & $\gg$ & +1.10 & +3.2 & 21 \\
\hline 16 & I $4 \quad 54 \quad 7$ & to 33.65 & - & $\circ$ & 3. & 8,8 & & $56 \quad 49.59$ & $9.5^{29 n}$ & +9 & 4 & I 3.2 & $.73 \mathrm{I}$ & +1.08 & +2.3 & 22 \\
\hline I 6 & $\searrow$ & - I 4.03 & + & 7 & I. 5 & 8,8 & 35 & $56 \quad 49.62$ & $\gg$ & +9 & 4 & $\times 4.0$ & $»$ & +1.08 & +2.3 & 23 \\
\hline 16 & $15 \quad 42 \quad 12$ & to 48.82 & + & I & 17.2 & I 2,8 & 35 & $57 \quad 4.76$ & $9.437 \mathrm{n}$ & +9 & 5 & 33.4 & 0.719 & +1.08 & +2.3 & 22 \\
\hline 16 & 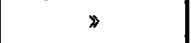 & -o 48.94 & + & 8 & 22.7 & I 2,8 & & $57 \quad 4.71$ & $»$ & +9 & 5 & 35.2 & , & +1.08 & +2.3 & 23 \\
\hline 19 & $\begin{array}{lll}15 & 21 & 37\end{array}$ & $-2 \quad 23.86$ & + & $\circ$ & 53.6 & 8,4 & 41 & $1954.5^{2}$ & $9.502 \mathrm{n}$ & $+I I$ & I & 0.2 & $0.71 \mathrm{I}$ & +1.06 & +1.0 & 24 \\
\hline 19 & $15 \quad 46 \quad 59$ & $-2 \quad 15.98$ & + & $\mathbf{I}$ & 30. & 8,4 & & $20 \quad 2.40$ & $9.453 n$ & $+1 I$ & I. 1 & 36.9 & 0.703 & +1.06 & +1.0 & 24 \\
\hline 20 & I $5 \quad 47 \quad 54$ & -034.73 & - & 4 & 2. & 16,8 & & $27 \quad 19.33$ & $9 \cdot 449 \mathrm{n}$ & + II & [ 37 & 0.5 & 0.697 & +1.06 & +0.6 & 25 \\
\hline $2 \mathrm{I}$ & I $533 \quad 55$ & $+I 23.46$ & - & 9 & 22.6 & 8,8 & 43 & $34 \times 8.62$ & $9.494 \mathrm{n}$ & +12 & 210 & 0.1 & 0.700 & +1.06 & +0.3 & 26 \\
\hline $2 \mathrm{I}$ & $\gg$ & -032.22 & + & 9 & 9.2 & 8,4 & & $34 \quad 18.13$ & $\gg$ & +12 & 29 & 56.6 & 》 & +1.06 & +0.3 & 27 \\
\hline 22 & I $538 \quad 16$ & +223.83 & - & 7 & 5.9 & 8,8 & 44 & $4 \mathrm{I} \quad 12.71$ & $9 \cdot 493 n$ & +12 & $24 I$ & $43 \cdot 5$ & 0.694 & +1.06 & 0.0 & 28 \\
\hline 22 & $》$ & $-2 \quad 5.75$ & - & 3 & $50 . x$ & 8,8 & 44 & $4 \mathrm{I} \quad 12.7^{\circ}$ & $\gg$ & +12 & $24 \mathrm{I}$ & $44 \cdot 5$ & " & +1.06 & 0.0 & 29 \\
\hline 24 & I $5 \quad 29 \quad 54$ & $+I 22.67$ & + & 3 & $3 \cdot 1$ & 8,4 & 45 & $5421.5^{I}$ & $9.52 O_{n}$ & +13 & 340 & 9.1 & $0.69 \mathrm{I}$ & +1.04 & -0.6 & 30 \\
\hline 24 & $\gg$ & $-0 \quad 59.85$ & - & $\mathbf{I}$ & 0.8 & 8,4 & & 5421.54 & $»$ & +13 & 340 & 7.6 & $»$ & +1.04 & -0.6 & 31 \\
\hline
\end{tabular}

Luglio 17 a 20 . Sereno splendido. La cometa fu vista qual macchia nebulosa informe, ampia meno di $I^{\prime}$ e debolissima così da essere puntata con difficoltà ed incertezza malgrado il bel sereno. A volte splendeva nel suo mezzo un nucleo simile alle ultime stelle percepibili nell' Amici, per cui, in complesso, si puo dire, che somigliava ad una stellina di ${ }^{2} \mathbf{m}_{5}$ fatta nebulosa sfocandola. Scompariva al primo chiarore dell' alba. - Luglio 2 I, 22 . Sereno fosco. Debolissima. Puntate incerte. - Luglio 25, 26. Sereno. Meno debole, la si può ragguagliare all' immagine di una stellina di I $^{\mathrm{m}}$ posta fuori di foco. - Luglio 29 . Sereno splendido. Aumentata di splendore, simile ad una stellina di $\mathrm{I}^{\mathrm{m}}$ sfocata. - Luglio 3I. Sereno impuro. - Agosto I. Sereno splendido. Cometa bene visibile ed ora le si puó assegnare $\mathrm{la} \mathrm{Io}^{\mathrm{m}}$, perchè sfocando le stelle di questa grandezza si ottengono immagini somiglianti ad essa. - Agosto 2, 3. Splendido, ma con Luna che è prossima al plenilunio e quindi rischiara tutta la notte. La cometa fu debole sia pel chiarore lunare, sia per quello susseguente dell' alba. Puntate incerte. L'effemeride Schulhof, in A. N. 4109 , indica che l'astro è prossimo al suo massimo splendore che, però, è vinto e soverchiato da quello lunare. La si deve abbandonare per qualche giono. - Agosto I 3 a 16 . Splendido. La si vede bene malgrado il residuo della luce lunare che, però, va ogni dì più scemando. Presentemente per avere un' apparenza simile alla cometa bisogna sfocare una stella di $9 \cdot 5$. - Agosto 19 a 24 . Periodo di sereno splendido. Essendo il cielo perfettamente puro, e senza Luna, la cometa fu vista nel suo maggior splendore che si ragguaglio ad una stella di $9^{\mathrm{m}}$ sfocata. - Dopo questo tempo si è dovuta lasciare per assenza dall' Osservatorio di Arcetri a tutto settembre, nè si potè riprenderla al ritorno, perchè ormai diventata invisibile per l'Amici.

Stelle di confronto.

\begin{tabular}{|c|c|c|c|c|c|c|c|}
\hline * & $\alpha 1906.0$ & $\delta 1906.0$ & Autorità & $*$ & $\alpha 1906.0$ & $d 1906.0$ & Autorità \\
\hline I & $23^{h} 45^{m} 40^{5}$ I 5 & $-13^{\circ} 37^{\prime} 59^{\prime \prime} 9$ & $\operatorname{Rad}_{3} 63^{6} 7$ & 4 & $23^{h} 5^{8^{m}} 3^{1} \cdot 79$ & - I $2^{\circ} 27^{\prime} 57^{\prime \prime} 8$ & $1 / 2$ (Sj. $9975+\mathrm{Par}_{2}$ \\
\hline 2 & 235 I II.I 5 & $\begin{array}{lll}-13 & \circ 2.8\end{array}$ & Schj. 9922 & 5 & $\begin{array}{lll}\circ & 5 & 34.84\end{array}$ & $\begin{array}{lll}-12 & 18 & 30.4\end{array}$ & $1 / 2\left(\mathrm{Sj} .28+\operatorname{Par}_{3} 87\right)$ \\
\hline 3 & $\begin{array}{lll}23 & 57 & 25.44\end{array}$ & $-1229 \quad 14.1$ & $1 / 3\left(W_{1}\right.$ II $\left.24+2 \mathrm{Sa}_{4} 2239\right)$ & 6 & $\circ$ I 254.25 & $\begin{array}{llll}- \text { II } & 44 & 16.0\end{array}$ & $1 / 3\left(W_{1} 170+2 \operatorname{Sa}_{3} 16\right)$ \\
\hline
\end{tabular}




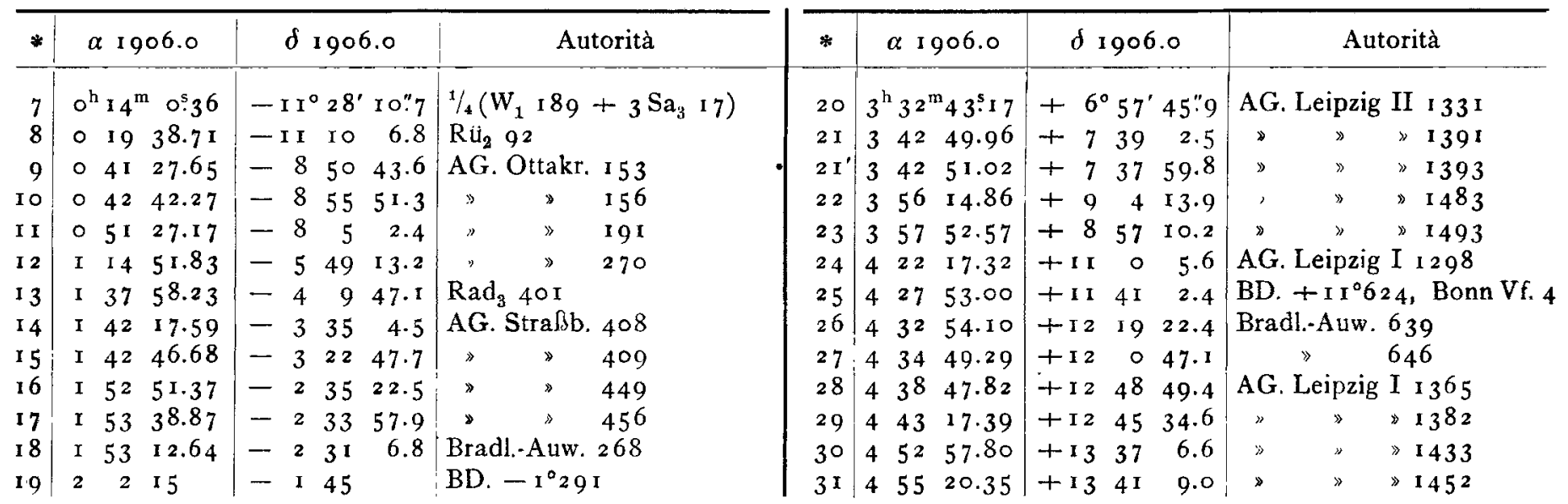

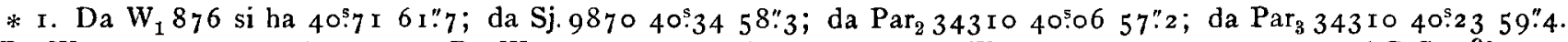

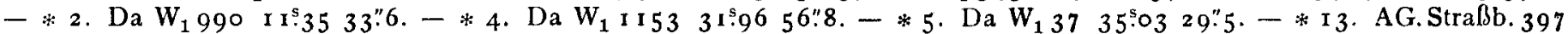

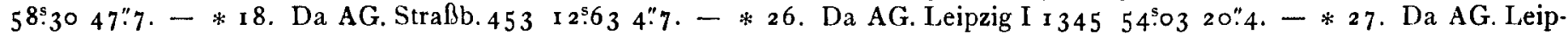
zig I I $353 \quad 49.26 \quad 47.7$.

Confronto $(\mathrm{O}-\mathrm{C})$ delle osservazioni coll' effemeride Schulhof in A. N. 4 rog.

\begin{tabular}{|c|c|c|c|c|c|}
\hline 1906 & $\Delta \alpha$ & $\Delta \delta$ & 1906 & $\Delta \alpha$ & $\Delta \delta$ \\
\hline Lugl. $17^{\mathrm{d}} \mathrm{o} 34$ I & -0.37 & +22.2 & Lugl. $26^{d}$ x $\circ 93$ & $-5^{5.05}$ & $-3 " .5$ \\
\hline 17.08995 & -0.24 & +16.7 & 29.07045 & -6.67 & -15.3 \\
\hline $18.0680 \mathrm{I}$ & -0.84 & +16.3 & 31.07075 & -8.28 & -22.1 \\
\hline $18.0893^{2}$ & -0.49 & $+21 \cdot 3$ & $3^{1.0935^{6}}$ & -7.70 & -20.9 \\
\hline 19.09317 & -0.98 & +9.9 & Agos. 1.06796 & -8.54 & -25.1 \\
\hline 19.09317 & $\rightarrow \mathrm{I} . \mathrm{OI}$ & +11.7 & 1.06796 & -8.67 & -24.4 \\
\hline 20.05814 & -1.56 & $+\mathrm{I2} \cdot 3$ & I. 11022 & -8.60 & -28.2 \\
\hline $21.073^{66}$ & -2.48 & +7.7 & 1. I 1022 & -8.77 & -26.0 \\
\hline 21.09149 & -1.95 & +10.5 & 2.10525 & -9.36 & -30.0 \\
\hline 22.08009 & -2.60 & +6.2 & 2.10525 & -9.18 & $-32 \cdot 5$ \\
\hline 25.09198 & -4.15 & $+\mathrm{I} .8$ & 2. I 1866 & -9.88 & -35.9 \\
\hline 25.09198 & -4.26 & +1.1 & 2.11866 & -9.63 & -35.6 \\
\hline 25.10614 & -4.28 & -1.1 & 3.11120 & - & - \\
\hline 25.10614 & -4.36 & -1.4 & I 3.07704 & $-\mathrm{I} 3.6 \mathrm{I}$ & -53.9 \\
\hline 26.09278 & $-5 \cdot 16$ & -5.1 & I 3.1 I 57 & -13.87 & -49.6 \\
\hline
\end{tabular}

\begin{tabular}{r|c|c}
\hline I906 & $\Delta \alpha$ & $\Delta \delta$ \\
\hline Agos.14.12114 & -13.02 & -63.6 \\
I4.12114 & -13.59 & -59.4 \\
16.09456 & -14.08 & -51.6 \\
I6.09456 & -14.05 & -50.8 \\
I6.12795 & -14.56 & -55.2 \\
I6.12795 & -14.61 & -53.4 \\
I9.11358 & -14.39 & -55.8 \\
I9.1312 & -14.17 & -57.7 \\
20.13182 & -13.03 & -53.0 \\
21.12208 & -13.74 & -47.2 \\
21.12208 & -14.23 & -50.7 \\
22.12507 & -13.45 & -52.1 \\
22.12507 & -13.46 & -51.1 \\
24.11920 & -13.70 & -47.3 \\
24.11920 & -13.67 & -48.8
\end{tabular}

Arcetri-Firenze, 1906 Dic. 20.

A. Abetti.

\section{Ausmessung des zweiten Sternhaufens im Hercules (Messier 92).}

Dieser Sternhaufen gehört zu den interessanten Gebilden, die von William Herschel Globular Clusters genannt worden sind. Von H. Schultz ist eine Anzahl Sterne dieses Objekts während der Jahre 1865-75 mit dem 9-zöll. Refraktor der Sternwarte in Upsala in sorgfältiger Weise mikrometrisch ausgemessen worden. Hauptsächlich aus diesem Grunde wurde dieses Objekt zur photographischen Ausmessung an der hiesigen Sternwarte aufgenommen. Eine erste Aufnahme des Objekts wurde am 29. April I 898 erhalten und zeichnet sich durch verhältnismäßig große Schärfe und Klarheit aus. Die Ausmessung umfaßt 348 Sterne und wurde zum größten Teil vom Assistenten cand. E. A. Neander bewerkstelligt. Die Reduktionen sind dann größtenteils von cand. $H$. $G$. Block und vom Assistenten Herrn $G$. B. Strömberg durchgeführt worden. Zur Vervollständigung des Materials sind noch von cand.
Block und vom Unterzeichneten die erforderlichen Supplementmessungen ausgefuhrt worden. Die Messungen wurden in je $z$ wei entgegengesetzten Lagen der Platte ausgefuhrt und dürften von konstanten Fehlern so gut wie frei sein.

Die Einzelheiten der Untersuchung sind in einer eben erscheinenden Abhandlung: $\gg$ Der zweite Sternhaufen im Hercules, Messier 92 " (Astron. iaktt. och undersökn. å Stockholms Observatorium Band 8 No. 3) enthalten, woselbst eine Note über zweckmäßige Berechnung der Refraktionskorrektionen noch als Anhang beigefügt worden ist.

Die Koordinaten des Sternhaufens sind:

$$
\begin{array}{ccc} 
& \alpha \text { I } 900.0 & \delta \text { I } 900.0 \\
\text { Messier } 92 & 17^{\mathrm{h}} \mathrm{I} 4^{\mathrm{m}} \cdot \mathrm{I} & +43^{\circ} \times 5^{\circ}
\end{array}
$$

derselbe ist also unweit von Messier I 3, dessen Koordinaten 